\title{
Engineering Alloplastic Temporomandibular Joint Replacements
}

\author{
Hani Sinno*, Youssef Tahiri, \\ Mirko Gilardino, Dennis Bobyn
}

\begin{abstract}
Temporomandibular disorders (TMD) are part of a heterogeneous group of pathologies that manifest with a constellation of signs and symptoms. They are the most frequent cause of chronic orofacial pain and are prevalent in $12 \%$ of the general population. Despite the debilitating nature of these disorders, there is no standardization for treatment of the diseased temporomandibular joint (TMJ). In this review, we present an overview of the functional anatomy of the TMJ and the engineering concepts that must be understood to better understand the indications for surgical management, the types of available treatments and the requirements for reconstruction. A comparison is made of the clinical outcomes with autogenous versus alloplastic reconstruction, including a history of alloplastic materials and the design features of currently available implants. Emphasis is made on material selection, modulus, stiffness, notch sensitivity and modularity. For the treatment of TMD, engineered TMJ alloplastic replacements have had considerable promise with additional room for improvement using new materials and recent design concepts.
\end{abstract}

KEYWORDS: Temporomandibular joint (TMJ), alloplastic, prosthetic, artificial, replacement, arthroplasty, reconstruction, engineering

\section{INTRODUCTION}

The temporomandibular joint (TMJ) is one of the most active joints in the human body. This unique "ball and socket" joint allows for more than 2000 hinge and sliding motions per day in activities such as talking, swallowing, and kissing. Temporomandibular disorders (TMD) are currently the most prevalent source of orofacial pain accounting for 12 percent of the adult population in the United States $(1,2)$. There is a strong $10: 1$ female predominance with the majority being between eighteen and fortyfive years of age (1-3). Congenital dysmorphism, trauma, osteoarthritis, rheumatoid arthropathy, ankylosis, condylar resorption, neoplasia, and previ-

\footnotetext{
*To whom correspondence should be addressed: Hani Sinno

Division of Plastic and Reconstructive Surgery, Department of Surgery, McGill University, Montreal, Qc, Canada, H3G 1A4. Email: hani. sinno@mail.mcgill.ca
}

ous failed reconstruction of the TMJ attribute to the development of TMD and are indications for TMJ reconstruction $(4,5)$. The presenting symptoms include headaches, changes in the mandibular range of motion, and pain $(1,6,7)$. Despite the significant psychosocial impact of TMD, the method of TMJ reconstruction remains controversial. Currently, autogenous and alloplastic joint replacements are available. The purpose of this review is to discuss the advantages and disadvantages of current TMJ replacements with an emphasis on engineering concepts and future improvements.

\section{TEMPOROMANDIBULAR DISORDERS}

Pathogenesis of degenerative TMJ diseases is thought to occur from the disequilibrium processes involving chondrocyte proliferation, differentiation, and degradation. Coupled with increasing 
inflammatory mediators, the hallmark of TMD is increased degradation of the joint tissue. Altered mechanical loading, female hormones, and alterations in the extracellular matrix of the TMJ are thought to cause TMD (8). Altered mechanical loading by either trauma or distorted function can surpass the healing capacity of the joint and cause irreversible damage $(9,10)$.

Traumatic injuries can also result in degenerative changes of the TMJ. Comminuted fractures of the mandibular condyle are difficult to treat and can lead to chronic pain and ankylosis. A study of 400 TMD patients found that a quarter of the cases were linked to a traumatic event (11). Whiplash injuries seemed to be especially linked to the development of painful jaw and the development of delayed TMD symptoms $(12,13)$.

In addition to mechanical etiology, genetic predisposition has also been linked to TMJ symptoms. The high prevalence of TMD in women has been correlated with estrogen and progesterone levels. In addition, the intensity of jaw pain has been associated with polymorphisms in the estrogen receptor (16). In addition to hormonal influences, genetic predisposition to TMD is also postulated to affect the joint extracellular matrix $(14,15)$.

Irrespective of the etiology of TMD, the symptoms can be devastating to the patients suffering from the disease. Consequently, the goals of TMJ reconstruction should be to: 1 . reduce patient suffering; 2 . improve joint function; 3 . reduce disability; 4. prevent morbidity; and 5. maintain acceptable treatment costs (17). To better understand how to achieve these goals, an understanding of the TMJ anatomy and function is required.

\section{ENGINEERING CONCEPTS OF THE TEMPORO- MANDIBULAR JOINT}

The TMJ can be simplified as a "ball and socket" joint. The mandibular condyle "ball" fits into the temporal bone mandibular fossa "socket" (Figure 1). It is capable of both rotational and translational motion. The ability of sliding on a hinge joint is unique to the TMJ. This allows for motion in various vector planes involved in speech, mastication, swallowing, and yawning (3).

This ball and socket lever system has an articular disc separating the mandible and the temporal bones. This avascular, insensate structure is attached to the condyle medially and laterally by collateral ligaments (Figure 1). Cartilage is found on the bony surfaces in the TMJ allowing for the dissipations of shearing and frictional loads generated by the functional jaw. Synovial fluid that bathes the TMJ acts as a vehicle for nutrient delivery and waste product removal. It also acts as a lubricant by reducing the coefficient of friction and the strain energy (18).

Functionally, the forces of mastication are distributed along the mandible. The tensile stresses develop at the sites of masseter muscle attachment to the bone which are converted to compressive stresses at the bite target. The forces are distributed (flow) through the stiffest components of the bone to the target point. This is the cortical part of the mandible ramus and body. At the target, the teeth and adjacent bone experience the maximal compressive stress. This stress continues to increase at the bite target until the geometry of the target changes (cleaving or crushing). The contralateral masseteric sling generates force that acts to stabilize the mandible from rotation. In addition to this TMJ hinge motion, the chewing and grinding of the found bolus require translational and sliding forces in the axial plane generated by the pterygoid muscles. Aberrant mechanical loading because of trauma or even changes from solid to soft diet

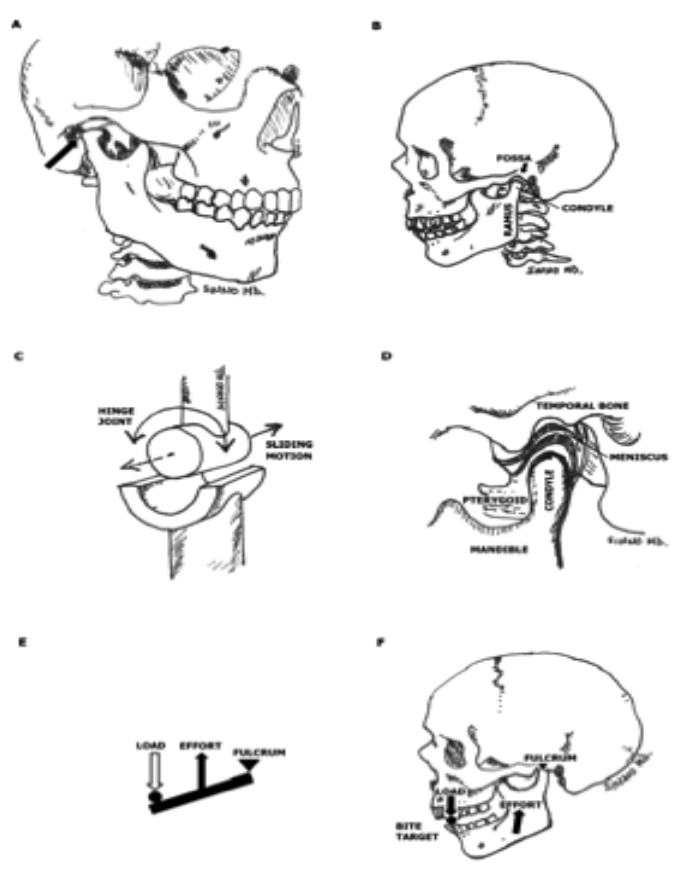

Figure 1.:A) Black arrow pointing to the anterolateral view of the TMJ as seen on a drawing of the human skull. B) Drawing of the left lateral TMJ on a human skull. C) A schematic of the TMJ as a hinge and sliding joint. D) Saggital section through the TMJ showing the meniscus and cartilage between the mandible and temporal bones of the TMJ. E) The jaw simplified as a third class lever: Lever: the mandible body; Fulcrum: the TMJ; Effort: force generated by the masseter muscle; and Load: the food bolus. F) Drawing of a lateral view of the human skull as a third class lever with a bite target as the load. 
can cause decreases in the thickness of the mandibular condylar cartilage and subchondral bone volume which can be reversed with the restoration of normal loading $(9,10,19)$. In addition, the forces generated with mastication can generate high joint reactive forces on the condylar cartilage. Accumulation of abnormal forces, trauma, and autoimmune reactions can wear the normal TMJ and lead to TMD. This can be a devastating, painful state that prevents normal jaw function and may also lead to bony resorption. TMJ reconstruction surgery can be an option to reverse these changes.

\section{RECONSTRUCTION OF THE TMJ AUTOGENOUS}

The initial management of TMD includes occlusal splints, physiotherapy, pharmacotherapy and complementary medicine. Surgery is offered as a last resort since it has been shown that the higher the number of prior jaw surgeries, the poorer the subjective outcome (20). Most authors agree however, that the main indications for joint replacement are the presence of a symptomatic severely damaged joint from either severe joint disease or failed previous surgeries.

The method of reconstruction of the TMJ with autogenous grafting versus alloplastic pros-

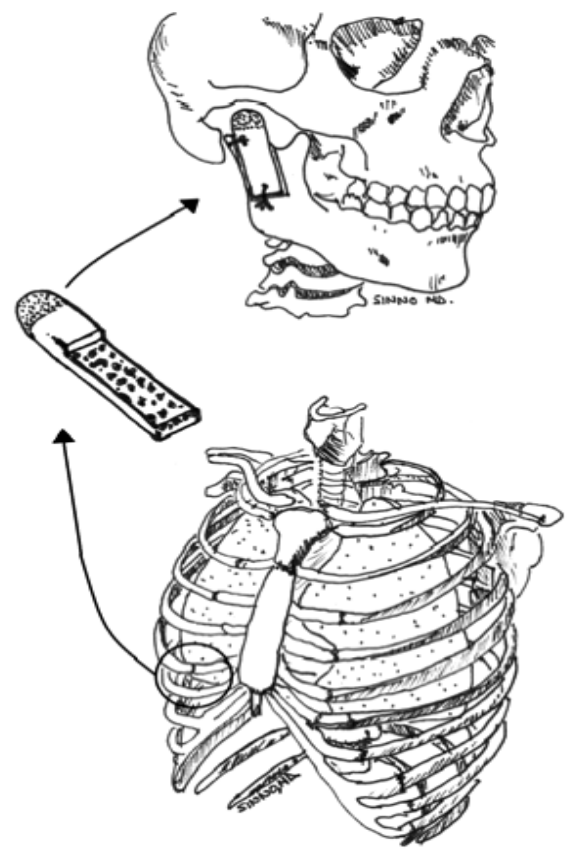

Figure 2: Drawing of the harvest of the costochondral rib graft for temporomandibular joint reconstruction. The decorticated rib graft is fit into the mandible as an inlay and fixed with $0.5 \mathrm{~mm}$ stainless steel wires. thetics is controversial. Many autogenous grafts and flaps have been described including fibula, metatarsal, sternoclavicular, iliac, and costochondral tissue (21). The most commonly used technique is the costochondral graft (Figure 2). This graft is unique in its biological compatibility, workability, functional adaptability, and minimal detriment to the patient. The growth potential of costochondral grafts is ideal in children. Cost has also been implicated as an advantage over alloplastic prosthetics. To our knowledge however, there are no cost analysis studies comparing the longer operative time and hospitalization required for patients undergoing autogenous TMJ reconstruction as compared with the initial high cost of alloplastic implants. Autogenous grafting for TMJ reconstruction has shown very satisfactory cosmetic and functional results. Complications do exist however, including donor site morbidity, facial and temporoparietal nerve injury, Frey's syndrome, recurrence of ankylosis, fracture, bone resorption, and unpredictable growth behaviour of the graft $(22,23)$. Another important consideration is that autogenous reconstruction only deals with the mandibular condyle portion "ball" of the joint. It does not address abnormalities of the temporal bone mandibular fossa "socket". Furthermore, when autogenous reconstruction was compared with alloplastic reconstruction of the TMJ, patients showed equal improvement in pain and jaw function (21). However, more patients required re-operation in the autogenous group $(66.67 \%$ : $n=18$ of $27)$, a third of who developed recurrent ankylosis (21). Autogenous grafting for TMJ reconstruction can be a satisfactory operation in the right patient with a known complication.

\section{ALLOPLASTIC}

Indications for alloplastic TMJ reconstruction are controversial. Mercuri has summarised the indications in his review: 1. Ankyolosis with severe anatomic abnormalities; 2. Failure of autogenous grafts; 3. Failure of Proplast-Teflon or Vitek-Kent or partial joints implants; 4 . Severe inflammatory joint disease that results in joint mutilation and functional disability (17). Unfortunately, due to the lack of current data, these criteria have been presented without the discussion of differences between available prosthetic options.

\section{History}

In light of the disadvantages in autogenous reconstruction, alloplastic TMJ reconstruction was increasing in popularity in the early 1980's. At that time, Vitek Inc. obtained FDA-approval for the use 
of their Proplast-Teflon implant designed for surgical replacement of dysfunctional TMJ. Proplast was developed by a chemical engineer, Dr. Charles Homsy, in the late 1960's. He combined this soft porous material with Teflon (polytetrafluoroethylene) resin to create a biomedical implant for TMJ replacement. The Proplast was used to encourage host tissue ingrowth and stability while the Teflon was designed to withstand wear from the joint. Homsy studied his materials in vitro, however he did not conduct wear nor animal testing of the Proplast disc implants (24). Despite incomplete testing (25) and warnings given by the supplier company Du Pont that "Teflon implants wore badly and had a tendency to disintegrate in load bearing joints" (26), it is estimated that 26,000 patients across North America were fitted with these implants. By the late 1980 's many patients presented with irreversible and life altering symptoms related to mechanical failure of the Vitek product. This was secondary to the implant failure under repeated loading causing soft tissue damage. On January 1991, the FDA ordered Vitek to remove its implants from the market.

\section{Implant Design}

Engineering Concepts:

To prevent repeating the Vitek implant catastrophe, thorough testing of materials for implant design must be conducted. In the world of total hip arthroplasty (THA), various materials have withstood the test of time for the past four decades. It would seem reasonable therefore to look at orthopaedic technology for better solutions to problems in craniofacial surgery. Choosing the appropriate materials, however, begins with understanding key engineering concepts and desirable implant properties.

Biocompatibility: The first tenet of implant design is to attain good fixation while causing the least amount of damage to the surrounding tissue. Modulus: The elastic modulus of a material is the measure of resistance to deformation for a given load or stress. For example, materials with lower elastic moduli or lower function stiffness (see below), are generally more load sharing with bone, a property that helps preserve native bone density and strength.

Stiffness: The stiffness of a material is dependent on both the elastic modulus and geometry of the device (equivalent to the arithmetic product of the two values). For two objects made of the same material, the larger the object the stiffer it is and thus, more resistant to deformation. One way to use relatively rigid metallic materials without nec- essarily creating an implant that is excessively stiff is to make the implant hollow or very porous. This design concept enables the fabrication of implants that better share load with bone (see below).

Stress Shielding: When different materials are placed adjacent to one another with a uniform load applied to both, the stiffer material will resist changes in deformation more than the more flexible material. For example, when a titanium metal plate (a higher modulus of elasticity than bone) is fixed adjacent to bone, stresses applied will be seen by the stiffer metal plate (Figure 3). Bone is said to be "stress shielded". According to Wolff's Law, bone will remodel according to in vivo loads; thus bone that is 'stress shielded' will lose density and consequently have less mass and strength in the case of revision surgery.

Notch Sensitivity: In addition to reducing stress shielding, promotion of bone ingrowth through porous metal coating will also increase implant fixation. The porous coating of hip implants has been shown to promote bone ingrowth (30-32). The ingrown bone rigidly fixes the implant and prevents pain and implant-host interface failure. The addition of a porous coating to the surface of implants however, causes stress concentrators that may propagate a fracture in notch sensitive material. Notch sensitivity is the degree to which the sensitivity of a material to fracture is enhanced by the presence of a surface inhomogeneity such as a notch created by surface porous coating. Notch sensitive materials are also prone to fracture at sudden changes in section, cracks, or scratches. In
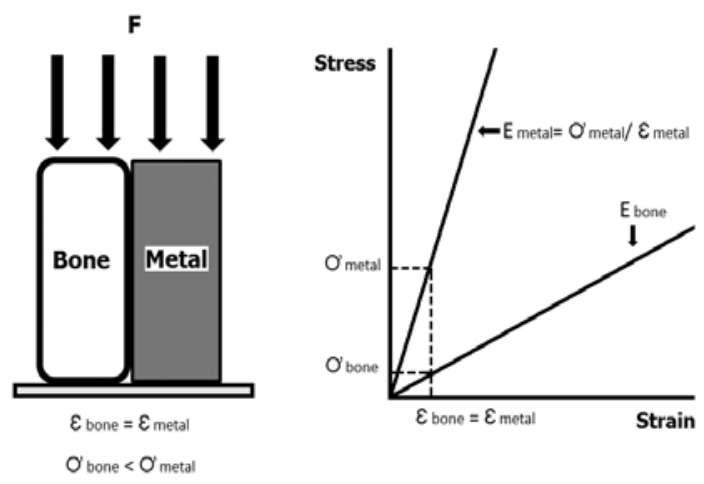

Figure 3: The load deformation curve on the right demonstrates that metal has a higher modulus than bone. More stress is required to deform metal than bone for a given strain. On the left, is a schematic of metal stress shielding bone for a given force F: Force; O': Stress; $\varepsilon$ : Strain= $(L 2-L 1) / L 1$, where $L 1$ is the initial length, $L 2$ is the length after deformation; $E$ : Modulus of Elasticity 
general, ductile materials are less notch sensitive than brittle materials.

Modularity: Modularity is another important concept in implant design, enabling the advantageous use of different materials for specific functions. For example, materials optimized for wear resistance can be used for the bearing components while materials that are less stiff can be used for load carrying and fixation to reduce stress shielding and consequent bone resorption.

Materials:

The same materials that are utilized in hip and knee arthroplasty are applicable to TMJ replacement. Cobalt-chromium and titanium alloys possess the necessary strength and fatigue resistance for implant stems and bodies, with titanium alloy having the advantage of lower elastic modulus and better load sharing characteristics. A variety of porous fixation surfaces are available for enhancing stability through tissue ingrowth. They include the more traditional beaded or fiber wire types of surface treatments and the newer higher porosity foam-like materials made of either titanium or tantalum (33-37). Potential bearing combinations include cobalt-chromium alloy against polyethylene or a hard-hard bearing using alumina oxide ceramic or cobalt-chromium alloy. Of significant note is the fairly recent development of highly cross-linked polyethylene, a very wear resistant formulation of ultra high molecular weight polyethylene that is witnessing widespread use in total hip and knee arthroplasty. Wear simulator studies have confirmed very low wear rates with these new materials (3740).

With the knowledge of implant design specifications, the appropriate component materials can be chosen. Among those, there is stainless steel. Such a material has appropriate ultimate tensile strength and fatigue properties. It should be avoided for TMJ implants however, as it is susceptible to crevice and inter-granular corrosion: in active areas such as the TMJ, this corrosion may lead to implant failure and consequent immune reaction and osteolysis. Stainless steel is not "biocompatible" under the loading conditions found in the TMJ. Cobalt-Chromium (CoCr) based alloys are biocompatible and unlike stainless steel are highly corrosion resistant. They have low notch sensitivity and thus can be treated with metal beads or fibre wire to promote bone ingrowth and significantly increase implant fixation (33). CoCr alloy metals have excellent wear properties and have stood the test of time in metal on metal hip implants for up to 20 years
(34). CoCr is very hard and abrasion resistant, but this property can be a disadvantage in TMJ implants. Due to its high elastic modulus, $\mathrm{CoCr}$ alloy implants cause significant stress shielding (35). Examples of implant migration and bone fracture secondary to the profound bone resorption have been reported in the orthopedic literature (35).

Titanium alloys are highly biocompatible with half the elastic modulus of CoCr. As a more flexible implant, it can more uniformly transfer loads and cause significantly less stress shielding to adjacent bone. Titanium however does not have good wear properties. When subjected to repetitive forces, it is fatigue resistant and thus breaks down. It should not be used at the joint interface. Furthermore, titanium is notch sensitive and thus should not be impregnated with beads or fibre wire to promote bony ingrowth. However, when the surface is roughened without notching, bone ongrowth occurs and thus improves fixation. Consequently, non-porous coated titanium alloy material can be used for the TMJ stem component to decrease stress shielding to the adjacent bone while still withstanding the forces generated at the TMJ. CoCr on the other hand has excellent wear resistance and is the reason why it is used as the femoral head component in total hip arthroplasty. It can withstand the high repetitive motion and forces up to three times body weight with acceptable wear properties lasting an estimated $20-30$ years. Consequently $\mathrm{CoCr}$ is an ideal material for the "ball" component of TMJ alloplastic implants.

Non-metallic materials have a significant role in joint replacement as well. Ceramics for example, are very stable and inert materials that can withstand high compressive forces. They are brittle however, and are susceptible to fracture when subjected to high tensile forces. They have been shown as useful alternatives as bearing material in highly loaded joints such as the hip.

Ultra-high molecular weight polyethylene (UHMWPE) has also had long term success in the orthopaedic literature as a joint spacer in the hip and knee (36). With repetitive loading however, it is subject to wear. To prevent particularization, osteolysis, and aseptic loosening, cross-linked ultra-high molecular weight polyethylene with improved wear resistance has been developed (37-39). Hip simulator studies have confirmed very low wear rates with these new materials $(38,40)$.

Contemporary metal-on-metal CoCr bearings have shown very low wear rates as compared to polyethylene. They have however been shown to release particles and ions from the articulating 
surfaces into the joint and the whole organism especially in the early phase after implantation. The released metal ions, especially chromium, are hypothesized to potentially trigger cytotoxic, carcinogenic and allergic reactions. The incidence of these implant-related complications is very low but long term data is unavailable. More research is required for the development of the best and safest bearing choice for implant design.

\section{CURRENT IMPLANTS}

Three prosthetic systems currently exist: 1. TMJ Implants (41); 2. TMJ Concepts (42); and 3. Biomet/Lorenz (43). Similar to hip implants, they are a "ball and socket" type of prosthetic joint. The three systems differ in their component materials (Table 1).

The condylar portion of all implants is made of $\mathrm{CoCr}$ alloy. The Biomet/Lorenz TMJ implants have a plasma spray titanium coating on the bonecontacting surface that allows bone apposition and biologic fixation. The $\mathrm{CoCr}$ bearing has high hardness and strength and attaches to the implant body with a modular taper connection.

Attached to the condylar component is the ramus which is made of $\mathrm{CoCr}$ alloy for the TMJ Implants and the Biomet/Lorenz system. The TMJ Concepts utilizes titanium alloy. Unlike the hip, the mandible is a thin bone that does not have much volume for implant fixation. The smaller modulus of titanium would better distribute load and decrease stress shielding in the inherently thin mandibular bone.

Connecting the "ball" to the "socket" is the fossa component. The TMJ Implants utilizes a metal-on-metal bearing made of $\mathrm{CoCr}$ alloy. Both the TMJ Concepts and the Biomet/Lorenz systems utilize UHMWPE for the fossa bearing material. Metal-on-metal components have been shown to have high wear resistance and long life expectancy in the hip. However, in the jaw where loads are relatively high and contact areas are smaller and less congruent than in the hip, there is potential for a more

\begin{tabular}{|l|l|l|l|}
\hline & Fossa & Condyle & Ramus \\
\hline TMJ Implants & Co-Cr-Mo Alloy & Co-Cr-Mo Alloy & Co-Cr-Mo Alloy \\
\hline TMJ Concepts & $\begin{array}{l}\text { Ti (UHMWPE } \\
\text { surface) }\end{array}$ & Co-Cr-Mo Alloy & Ti Alloy \\
\hline Biomet/Lorenz & UHMWPE & $\begin{array}{l}\text { Co-Cr-Mo Alloy } \\
\text { (Ti surface) }\end{array}$ & Co-Cr-Mo Alloy \\
\hline
\end{tabular}

Table 1: Components of the three TMJ prosthetic systems. Co: cobalt; Cr: Chromium; Mo: molybdenum; Ti: titanium; UHMWPE: ultra-high molecular weight polyethylene aggressive wear environment due to higher contact stresses. This is equally true for the CoCr-UHMWPE bearing components of the TMJ Concepts and Biomet/Lorenz systems. All three systems utilize titanium alloy screw for implant fixation to bone. The current available data for all implants have been promising. In 2004 Mercuri and Giobbie-Hurder showed high success rates with TMJ Concepts systems with a follow-up period of 60 months (44). Saeed and Speculand independently reviewed their use of the TMJ Implants on a total of 154 patients with acceptable outcomes $(21,45)$. The Biomet/ Lorenz system was reviewed in a case series describing the manufacturer's results (46). The objective outcomes of jaw function were shown to be highest in the multiply operated patients (3). These patients had poorer subjective responses however, which was attributed to psychological factors (3). Moreover, total alloplastic TMJ replacements have shown promising treatment outcomes reporting decreased patient pain and improved jaw function.

Alloplastic TMJ reconstruction devices have shown considerable predictability and reliability. Many advantages exist over autogenous grafts: 1. Immediate jaw motion and physiotherapy; 2 . No need for secondary donor site; 3 . Custom made alloplastic implant capability. The immediate capability for patients to undergo physiotherapy is perhaps the most important advantage of alloplastic implants. The patient's mandible is able to immediately function after implantation and thus continuous active and passive muscle activity allows for improved outcome and decreased stiffness of the joint. The decrease in secondary donor site morbidity is also a very attractive option over autogenous grafting. Not only does it prevent complications to other sites of the body, but operative time and costs are also decreased. Alloplastic materials can also be custom made to mimic the normal anatomic contours of the structures they are to replace. They can therefore be better stabilized and allow for faster recovery time.

Despite the many advantages to alloplastic prosthetics, there are known limitations. Alloplastic prosthetics are contraindicated in children, non-informed patients, in patients with uncontrolled systemic disease, in patients allegic to these materiels, and finally in patients with an active infection at the site of implantation (17). They should not be used in children as the implants may interfere with the normal growth of the facial skeleton and they would need to be replaced when facial maturity is reached. Another limitation of alloplastic prosthetics is their initial implant cost. The relative novelty of 
the modern TMJ implants limits the long-term data regarding material wear, failure, and implant stability.

\section{FUTURE DIRECTION / CONCLUSION}

Generalization of the improvements in patients undergoing total alloplastic TMJ replacement is limited to the low number of studies and the few surgeons and manufactures available. New technologies have been described in different fields that can improve current TMJ implants. For example, the use of cross-linked UHMWPE for the TMJ fossa may prove superior in wear to the current available implant materials. In addition, bisphosphanates are a group of anti-osteoclastic agents that have an anti-resorptive effect used in osteopenic patients for reducing bone resorption, increasing bone density, and decreasing fracture incidence. Studies have showed maintenance of bone mineral density around bisphosphanate impregnated hip implants $(47,48)$. The same technology may be applied to the TMJ implants to counter the stress shielding effects on the mandible. Another promising field advancing TMJ reconstruction technology is tissue engineering. Recent studies have manipulated rat bone marrow mesenchymal stem cells to form the shape and dimensions of a human mandibular condyle $(49,50)$. Such technology may provide anatomic-specific autogenous tissue implantation with the potential to adapt to the functional loads in the TMJ. Moreover, long-term clinical studies and improved implant design can potentially further improve the current available technology and help the millions of patients suffering from TMD disorders.

To conclude, the goal of TMJ reconstruction should appropriately address the presenting complaints of the patients without causing harm. Both autogenous and alloplastic TMJ reconstruction are available surgical options. Autogenous reconstruction is indicated in children as these grafts have growth potential. Modern alloplastic TMJ reconstruction has also shown improved subjective and objective outcomes with fewer complications than autogenous grafting. Long-term data however are insufficient to appropriately predict the life expectancy of the alloplastic systems. With understanding of the functional anatomy of the jaw and the engineering concepts and mechanics of the TMJ, superior designs and long-term studies can improve the systems currently available.

\section{REFERENCES}

1. LeResche L. Epidemiology of temporomandibular disorders: implications for the investigation of etiologic factors. Crit Rev Oral Biol Med 1997: 8: 291-305.

2. Lipton JA, Ship JA, Larach-Robinson D. Estimated prevalence and distribution of reported orofacial pain in the United States. J Am Dent Assoc 1993: 124: 115-21.

3. Guarda-Nardini L, Manfredini D, Ferronato G. Temporomandibular joint total replacement prosthesis: current knowledge and considerations for the future. Int $\mathrm{J}$ Oral Maxillofac Surg 2008: 37: 103-10.

4. Obeid G, Guttenberg SA, Connole PW. Costochondral grafting in condylar replacement and mandibular reconstruction. J Oral Maxillofac Surg 1988: 46: 177-82.

5. Mercuri LG. The use of alloplastic prostheses for temporomandibular joint reconstruction. J Oral Maxillofac Surg 2000: 58: 70-5.

6. Gelb H, Bernstein IM. Comparison of three different populations with temporomandibular joint pain-dysfunction syndrome. Dent Clin North Am 1983: 27: 495-503.

7. McNeill C. Management of temporomandibular disorders: concepts and controversies. J Prosthet Dent 1997: 77: 510-22.

8. Wadhwa S, Kapila S. TMJ disorders: future innovations in diagnostics and therapeutics. J Dent Educ 2008: 72: 93047.

9. Kiliaridis S, Thilander B, Kjellberg H, Topouzelis N, Zafiriadis A. Effect of low masticatory function on condylar growth: a morphometric study in the rat. Am J Orthod Dentofacial Orthop 1999: 116: 121-5.

10. Ravosa MJ, Kunwar R, Stock SR, Stack MS. Pushing the limit: masticatory stress and adaptive plasticity in mammalian craniomandibular joints. J Exp Biol 2007: 210: 628-41.

11. De Boever JA, Keersmaekers K. Trauma in patients with temporomandibular disorders: frequency and treatment outcome. J Oral Rehabil 1996: 23: 91-6.

12. Carroll LJ, Ferrari R, Cassidy JD. Reduced or painful jaw movement after collision-related injuries: a populationbased study. J Am Dent Assoc 2007: 138: 86-93.

13. Sale $H$, Isberg $A$. Delayed temporomandibular joint pain and dysfunction induced by whiplash trauma: a controlled prospective study. J Am Dent Assoc 2007: 138: 1084-91.

14. Abubaker AO, Raslan WF, Sotereanos GC. Estrogen and progesterone receptors in temporomandibular joint discs of symptomatic and asymptomatic persons: a preliminary study. J Oral Maxillofac Surg 1993: 51: 1096-100.

15. Milam SB, Aufdemorte TB, Sheridan PJ, et al. Sexual dimorphism in the distribution of estrogen receptors in the temporomandibular joint complex of the baboon. Oral Surg Oral Med Oral Pathol 1987: 64: 527-32.

16. Kang SC, Lee DG, Choi JH, et al. Association between estrogen receptor polymorphism and pain susceptibility in female temporomandibular joint osteoarthritis patients. Int J Oral Maxillofac Surg 2007: 36: 391-4. 
17. Mercuri LG. Alloplastic temporomandibular joint reconstruction. Oral Surg Oral Med Oral Pathol Oral Radiol Endod 1998: 85: 631-7.

18. Jay GD, Torres JR, Warman ML, Laderer MC, Breuer KS. The role of lubricin in the mechanical behavior of synovial fluid. Proc Natl Acad Sci U S A 2007: 104: 6194-9.

19. Bouvier M. Effects of age on the ability of the rat temporomandibular joint to respond to changing functional demands. J Dent Res 1988: 67: 1206-12.

20. Mercuri LG. Subjective and objective outcomes in patients reconstructed with a custom-fitted alloplastic temporomandibular joint prosthesis. J Oral Maxillofac Surg 1999: 57: 1427-30.

21. Saeed N, Hensher R, McLeod N, Kent J. Reconstruction of the temporomandibular joint autogenous compared with alloplastic. Br J Oral Maxillofac Surg 2002: 40: 296-9.

22. Politis $\mathrm{C}$, Fossion $\mathrm{E}$, Bossuyt $\mathrm{M}$. The use of costochondral grafts in arthroplasty of the temporomandibular joint. J Craniomaxillofac Surg 1987: 15: 345-54.

23. Figueroa AA, Gans BJ, Pruzansky S. Long-term follow-up of a mandibular costochondral graft. Oral Surg Oral Med Oral Pathol 1984: 58: 257-68.

24. Homsy CA. Bio-compatibility in selection of materials for implantation. J Biomed Mater Res 1970: 4: 341-56.

25. Kent JN. An important lesson about biomaterials in the TMJ. J Oral Maxillofac Surg 1991: 49: 442-3.

26. Paul A. Magnuson CJ. The Temporomandibular Joing Implants Products Liability Litigation. October 19, 2005: No. 95-1394.

27. Skinner HB, Curlin FJ. Decreased pain with lower flexural rigidity of uncemented femoral prostheses. Orthopedics 1990: 13: 1223-8.

28. Vresilovic EJ, Hozack WJ, Rothman RH. Incidence of thigh pain after uncemented total hip arthroplasty as a function of femoral stem size. J Arthroplasty 1996: 11: 304-11.

29. Wright JM, Pellicci PM, Salvati EA, et al. Bone density adjacent to press-fit acetabular components. A prospective analysis with quantitative computed tomography. J Bone Joint Surg Am 2001: 83-A: 529-36.

30. Robertson DM, Pierre L, Chahal R. Preliminary observations of bone ingrowth into porous materials. J Biomed Mater Res 1976: 10: 335-44.

31. Pilliar RM, Cameron HU, Macnab I. Porous surface layered prosthetic devices. Biomed Eng 1975: 10: 126-31.

32. Klika AK, Murray TG, Darwiche H, Barsoum WK. Options for acetabular fixation surfaces. J Long Term Eff Med Implants 2007: 17: 187-92.

33. Luckey HA, Lamprecht EG, Walt MJ. Bone apposition to plasma-sprayed cobalt-chromium alloy. J Biomed Mater Res 1992: 26: 557-75.

34. Heisel C, Thomsen M, Jakubowitz E, Kretzer JP. [Particle release in metal-on-metal bearings. A risk analysis]. Orthopade 2008: 37: 644-9.
35. Bobyn JD, Glassman AH, Goto $\mathrm{H}$, et al. The effect of stem stiffness on femoral bone resorption after canine porouscoated total hip arthroplasty. Clin Orthop Relat Res 1990: 196-213.

36. Galvin A, Brockett C, Williams S, et al. Comparison of wear of ultra-high molecular weight polyethylene acetabular cups against surface-engineered femoral heads. Proc Inst Mech Eng [H] 2008: 222: 1073-80.

37. McKellop H, Shen FW, Lu B, Campbell P, Salovey R. Development of an extremely wear-resistant ultra high molecular weight polyethylene for total hip replacements. J Orthop Res 1999: 17: 157-67.

38. Pedersen DR, Brown TD, Hillis SL, Callaghan JJ. Prediction of long-term polyethylene wear in total hip arthroplasty, based on early wear measurements made using digital image analysis. J Orthop Res 1998: 16: 557-63.

39. Wang A. Wear of ultra-high molecular weight polyethylene acetabular cups in a physiological hip joint simulator in the anatomical position using bovine serum as a lubricant. Proc Inst Mech Eng [H] 1998: 212: 71-2; author reply 72-3.

40. Bistolfi A, Turell MB, Lee YL, Bellare A. Tensile and tribological properties of high-crystallinity radiation crosslinked UHMWPE. J Biomed Mater Res B Appl Biomater 2008.

41. TMJ IMPLANTS INCORPORATED. http://wwwtmjcom/.

42. TMJ CONCEPTS. http://tmjconceptscom/.

43. BIOMET MICROFIXATION. http://wwwlorenzsurgicalcom/.

44. Mercuri LG, Giobbie-Hurder A. Long-term outcomes after total alloplastic temporomandibular joint reconstruction following exposure to failed materials. J Oral Maxillofac Surg 2004: 62: 1088-96.

45. Speculand B, Hensher R, Powell D. Total prosthetic replacement of the TMJ: experience with two systems 19881997. Br J Oral Maxillofac Surg 2000: 38: 360-9.

46. Quinn P. Lorenz Prosthesis. Oral Maxillofacial Surgery Clinics North America 2000: 12: 93-104.

47. Venesmaa PK, Kroger HP, Miettinen HJ, et al. Alendronate reduces periprosthetic bone loss after uncemented primary total hip arthroplasty: a prospective randomized study. J Bone Miner Res 2001: 16: 2126-31.

48. Wilkinson JM, Stockley I, Peel NF, et al. Effect of pamidronate in preventing local bone loss after total hip arthroplasty: a randomized, double-blind, controlled trial. J Bone Miner Res 2001: 16: 556-64.

49. Alhadlaq A, Mao JJ. Tissue-engineered neogenesis of human-shaped mandibular condyle from rat mesenchymal stem cells. J Dent Res 2003: 82: 951-6.

50. Mao JJ, Giannobile WV, Helms JA, et al. Craniofacial tissue engineering by stem cells. J Dent Res 2006: 85: 966-79. 
Hani Sinno (B.Sc., M.Eng., M.D.,C.M.) is in his forth year of plastic surgery residency at McGill University. He was a junior editor and then a senior editor for the McGill Journal of Medicine during his medical training at McGill University Medical School. His research interests include reconstructive surgery, and biomedical engineering.

Youssef Tahiri (M.D.,C.M.) is currently in his second year of residency in plastic surgery at McGill University.

Mirko Giladino (B.Sc., M.Sc., M.D.,C.M.) is currently a staff Surgeon in plastic surgery at McGill University. He is the Director of the Pediatric Craniofacial Program at the Montreal Childrens Hospital. His research focus is on craniofacial surgery as well as pediatric plastic surgery.

Dennis Bobyn (PhD) is currently Director of the Orthopedic Research Laboratories at the Montreal General Hospital. His research interests are novel joint replacement technologies. 